\title{
Frustration and the Kondo effect in heavy fermion materials
}

\author{
Piers Coleman ${ }^{1,2}$ and Andriy H. Nevidomskyy ${ }^{1,3}$ \\ ${ }^{1}$ Center for Materials Theory, Department of Physics and Astronomy, \\ Rutgers University, Piscataway, N.J. 08854, USA \\ ${ }^{2}$ Department of Physics, Royal Holloway, \\ University of London, Egham, Surrey TW20 OEX, UK and \\ ${ }^{3}$ Department of Physics and Astronomy, Rice University, Houston, Texas 77005, USA
}

(Dated: November 1, 2018)

The observation of a separation between the antiferromagnetic phase boundary and the small-large Fermi surface transition in recent experiments has led to the proposal that frustration is an important additional tuning parameter in the Kondo lattice model of heavy fermion materials. The introduction of a Kondo (K) and a frustration (Q) axis into the phase diagram permits us to discuss the physics of heavy fermion materials in a broader perspective. The current experimental situation is analysed in the context of this combined "QK" phase diagram. We discuss various theoretical models for the frustrated Kondo lattice, using general arguments to characterize the nature of the $f$-electron localization transition that occurs between the spin liquid and heavy Fermi liquid ground-states. We concentrate in particular on the Shastry-Sutherland Kondo lattice model, for which we establish the qualitative phase diagram using strong coupling arguments and the large- $N$ expansion. The paper closes with some brief remarks on promising future theoretical directions. 


\section{INTRODUCTION}

Since the 1980s, the Doniach scenario for heavy fermion behaviour $\underline{1}$, with a single quantum critical point linking the antiferromagnet and the heavy fermion metal, has provided the central conceptual framework for the understanding of heavy fermion materials. With the growth of interest in quantum criticality it has been tacitly assumed that the single magnetic quantum critical point (QCP) predicted by Doniach must uniquely describe the antiferromagnetic quantum criticality ${ }^{2}, \frac{3}{2}$ seen in heavy fermion metals.

Today however, there is a growing sense that the Doniach scenario for heavy fermion behaviour may be insufficiently flexible to account for the body of non-Fermi liquid (NFL) behaviour seen in heavy fermion materials. In particular, recent studies of $\mathrm{YbRh}_{2} \mathrm{Si}_{2}$ under pressure $\stackrel{4}{\underline{4}}$ and doping with Co and $\mathrm{Ir}^{\underline{5}}$ or $\mathrm{Ge}^{\underline{6}}$, point to the existence of two different transitions as a function of magnetic field - an antiferromagnetic (AFM) quantum critical point (QCP) on one hand, and the small-to-large Fermi surface crossover on the other, manifested by an abrupt jump in the Hall resistivity. While the position of the antiferromagnetic transition depends on pressure and doping, the small-to-large Fermi surface transition is unaffected by changes in pressure and doping ${ }^{\underline{5}}$. In another heavy fermion compound, YbAgGe, several magnetic transitions are observed as a function of applied magnetic field, and crucially, the non-Fermi liquid (NFL) state occupies a finite range of magnetic field, $5<H<10 \mathrm{~T}$, separating the AFM and the heavy Fermi liquid phases ${ }^{7}$.

These experiments suggest that under certain conditions, the transition between the fully developed antiferromagnet and the heavy fermion metal involves two distinct quantum critical points (QCPs), or possibly even a line of fixed points forming a quantum critical phase, realized in YbAgGe and doped $\mathrm{YbRh}_{2} \mathrm{Si}_{2}$ as a function of magnetic field ${ }^{5.7}$ and perhaps in $\beta-\mathrm{YbAlB}_{4}$ as a function of pressure $\stackrel{89}{9}$. By contrast, Doniach's classic phase diagram for heavy fermion physics in the Kondo lattice describes a direct second-order phase transition from an AFM phase into a heavy Fermi liquid as the strength of the Kondo coupling between the conduction and $f$-electrons increases. While such direct transitions have been observed, for example, in $C e C u_{6-x} A u_{x} \frac{10,11}{1}$ and in $C e N i_{2} G e_{2} \frac{12}{\text {, }}$ the more recent results indicate that the single-step transition is not universal. This line of reasoning leads us to conclude that that the phase space of the Kondo lattice must involve additional variables beyond the strength of the Kondo coupling $(K)$.

The simplest and most intriguing possibility is that the global magnetic phase diagram for heavy fermions requires an additional axis $\underline{13}-\underline{18}$, the ' $Q$ ' axis, which measures the additional quantum zeropoint motion of the spins induced by magnetic frustration. 


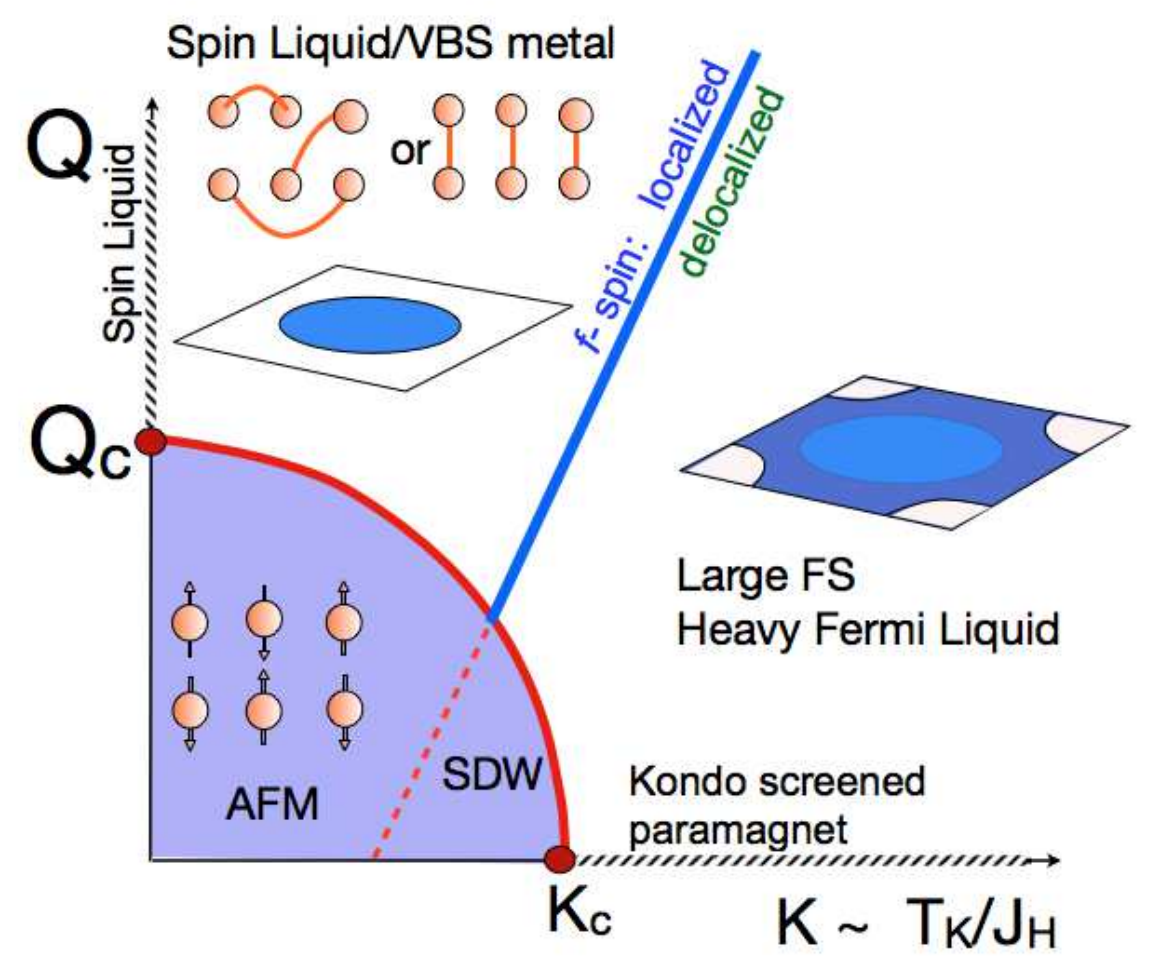

Figure 1: (Colour online) Schematic phase diagram of the Kondo lattice model in the parameter space of the Doniach axis $T_{K} / J^{H}$ and the quantum frustration axis $Q=1 / S$. A common antiferromagnetic phase boundary stretches from $K=K_{c}$ on the Kondo axis to $Q=Q_{c}$ on the frustration axis. At large $Q$, small $K$, a spin-liquid metal with localized $f$-electrons and a small Fermi surface forms, whereas at large $K$, a heavy Fermi liquid with a large Fermi surface and delocalized $f$-electrons develops. Since the volume of the Fermi surface is conserved, it is not possible, on a variety of lattices, to evolve smoothly from a small to a large Fermi surface, so that the spin liquid metal and the heavy Fermi liquid must be separated by a zero-temperature quantum phase transition.

In this paper we explore and review this idea. The two-axis diagram describing the joint effects of the Kondo screening $(K)$ and quantum zero-point motion $(Q)$ we call the " $Q K$ " diagram, shown in its simplest form in Fig 1. To illustrate the idea, first consider draining away the mobile electrons in a heavy fermion material to reveal the underlying magnetic lattice of $f$-electrons, $(K=0)$, each forming local moments coupled together via short-range antiferromagnetic (AFM) Heisenberg interactions of characteristic strength $J^{H}$. We then reintroduce the mobile electrons, and consider the effect of tuning up their coupling $K$ to the underlying magnetic lattice, as illustrated in Fig. 2

The new element, is that this lattice of local moments is considered to be magnetically frustrated. 
The appearance of frustration in real heavy fermion systems may take various guises - in certain cases, it can appear as direct geometric frustration, as in the pyrochlore heavy fermion material $\mathrm{Pr}_{2} \mathrm{Ir}_{2} \mathrm{O}_{7} \underline{19}$ and the Shastry-Sutherland lattice compound, $\mathrm{Yb}_{2} \mathrm{Pt}_{2} \mathrm{~Pb}^{20}$. Frustration can also take other forms, derived from competing interactions of various kinds. For example, in the "heavy fermion" physics of bilayer He-3, frustration may derive from ring-exchange effects in the lower, almost localized layer of helium atoms 21 .

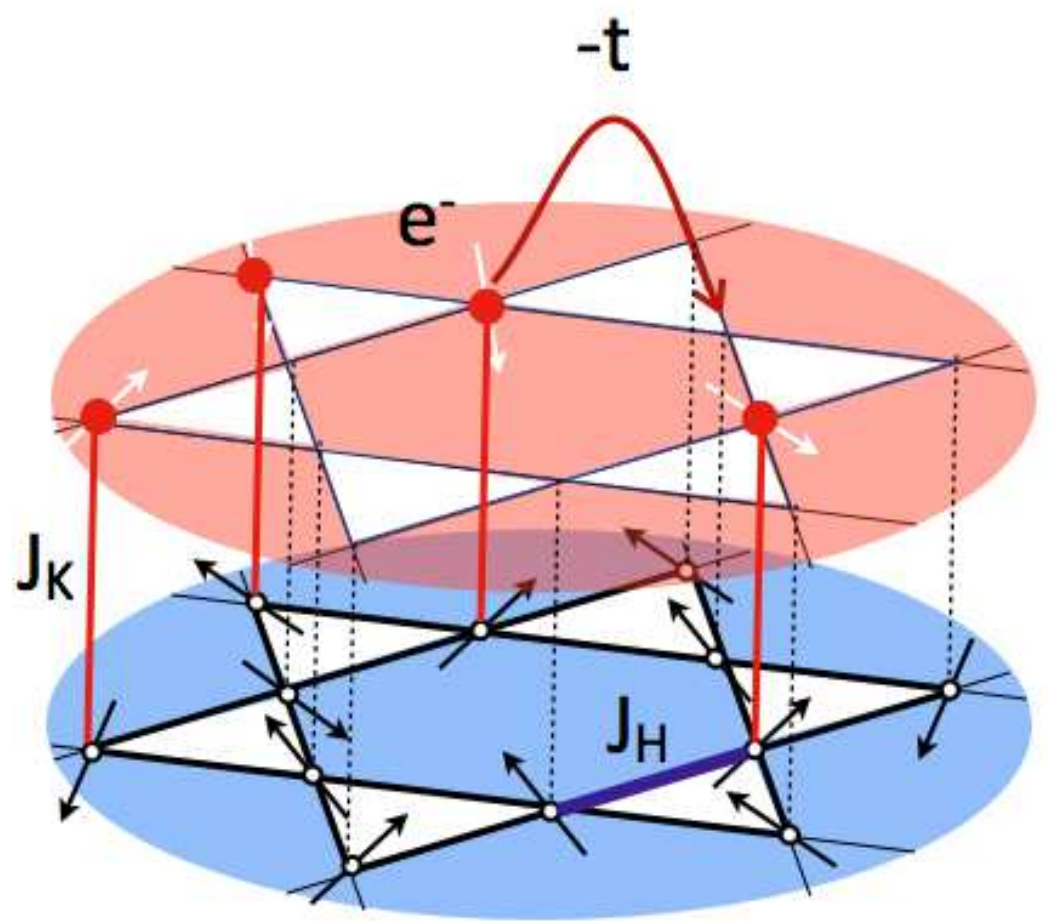

Figure 2: (Colour online) Illustrating the Kondo coupling $\left(J_{K}\right)$ between mobile electrons and an underlying frustrated spin system in a hypothetical Kondo-Kagomé lattice.

The staggered order-parameter of a quantum antiferromagnet $\vec{S}_{\mathbf{Q}}$ does not commute with the Hamiltonian, giving rise to zero-point fluctuations in the magnetization. In stable antiferromagnets these fluctuations act to reduce the size of the local moment below its classical value $\underline{22}$, but by increasing the frustration, the zero point motion can be driven so high that at some critical value $Q=Q_{c}$, the antiferromagnetic order melts, forming a spin liquid or a valence bond solid of spin dimers $\frac{23,24}{2}$. Depending on the lattice, this tuning can be done in various ways as illustrated in 
Fig. 3, For linguistic convenience, we shall loosely refer to this region as the "spin-liquid region" of the $Q K$ diagram.

Antiferromagnetic order can also be destroyed by the screening effects of the Kondo physics. According to the Doniach scenario, if reintroduce the electrons to the Kondo lattice, once the Kondo temperature becomes comparable with the RKKY coupling between the moments, $K \equiv$ $T_{K} / J^{H}=K_{c} \sim 1$, the screening of the local moments becomes complete, and a quantum phase transition into a heavy Fermi liquid takes place. In the $Q K$ diagram, this is the limiting behaviour along the $x$-axis $(Q=0)$, where frustration is absent.

The key idea of the $Q K$ diagram is to unify the Kondo and frustration effects as shown in Fig. 1, in which the two limiting quantum critical points at $Q=Q_{c}$ and $K=K_{c}$ are linked by a single antiferromagnetic phase boundary. Outside this phase boundary, at large $K>K_{c}$ but small $Q$, the system is a heavy Fermi liquid, in which the local magnetic moments are fully screened, donating their spin degrees of freedom to the Fermi sea to form a large Fermi surface of heavy electrons. By contrast, at small $K$ and larger than critical frustration $Q>Q_{c}$, the localized spins form a metallic spin liquid (or valence bond solid), and the conduction electrons are decoupled from the spin fluid, forming a metal with a small Fermi surface. Since the size of the Fermi surface is an adiabatic invariant, this leads us to conclude that there is no continuous way to move from the spin liquid metal to the heavy Fermi liquid. This leads to the tentative conclusion that at the very least, there must be one or more zero-temperature phase transitions separating the heavy Fermi liquid from the spin liquid metal.

In this paper we examine this reasoning in greater detail in relationship both to concrete theoretical models and real materials. One of the unexpected surprises, is that the phase boundary between the spin liquid and the heavy Fermi liquid is only guaranteed to exist in lattices where the unit cell contains an odd number of local moments. At the end of the article we discuss the relationship of the $Q K$ diagram to current experiments and ongoing efforts to theoretically understand the nature of heavy fermion quantum criticality.

\section{THE QK DIAGRAM.}

\section{A. Kondo Screening vs Zero-point motion}

One of the main ideas of $Q K$ phase diagram, is the existence of two distinct mechanisms for the destruction of antiferromagnetism in a Kondo lattice. We begin with a discussion of this idea. If 
(a)

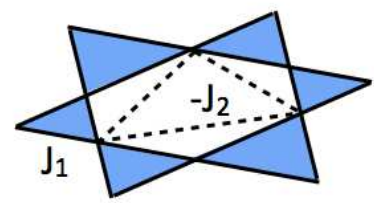

(b)

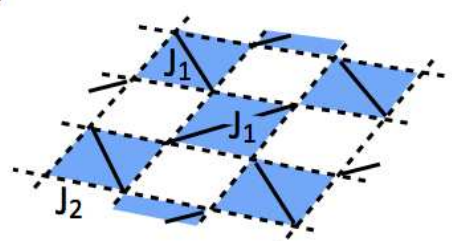

(c)

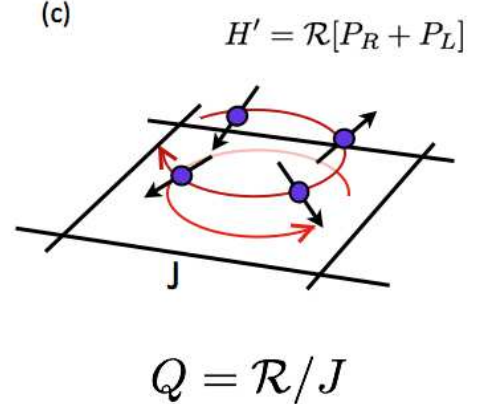

Figure 3: (Colour online) Frustrated Heisenberg models with a tunable degree of frustration $Q$. (a) The geometrically frustrated $S=1 / 2$ kagomé lattice, with next nearest neighbour ferromagnetic couplings to stabilize antiferromagnetism, and (b) the Shastry-Sutherland model, with alternating diagonal antiferromagnetic interactions $J_{1}$ on the a checker-board array and a nearest neighbour interaction of strength $J_{2}$. For (a) and (b), when $Q=J_{1} / J_{2}$ is large, the antiferromagnetism is destroyed, leading to a spin liquid in the kagomé lattice, and a valence bond solid across the diagonal bonds in the Shastry-Sutherland lattice, respectively. (c) The $2 \mathrm{D}$ Heisenberg antiferromagnet with a spin ring exchange term ${ }^{25}$ Eq. (2). When $Q=\mathcal{R} / J$ is increased, the two-sublattice magnetization is destroyed.

we drain the electrons out of a Kondo lattice, we are left with a system of localized moments that interact with each other via an arbitrary Heisenberg interaction $J^{H}$, plus perhaps the ring-exchange terms:

$$
\hat{H}_{f}=\sum J_{i j}^{H} \hat{\mathbf{S}}_{i} \cdot \hat{\mathbf{S}}_{j}+\hat{H}_{\square}
$$

In some cases it may be necessary to consider an additional spin ring exchange effect:

$$
\hat{H}_{\square}=\mathcal{R} \sum_{\text {plaquettes }}\left[\hat{P}_{R}+\hat{P}_{L}\right]
$$

where $\mathcal{R}$ is the amplitude for ring exchange of spins around a plaquette, and $\hat{P}_{R, L}$ are the operators that exchange spins in a right, or left-handed sense around the plaquette.

In heavy fermion systems the localized moments develop in partially filled $4 f$ or $5 f$ shells. Unlike the classic examples of magnetic frustration, heavy fermion materials are metals, and hybridization with conduction electrons plays crucial role. In well-localized heavy electron systems, the hybridization between $f$ - and conduction electrons gives rise to a Kondo (antiferromagnetic) on-site interaction $J_{K}$ between the spins of the conduction and $f$-electrons. Once we re-introduce 
conduction electrons, the combined Hamiltonian becomes

$$
\hat{H}=\hat{H}_{f}+\hat{H}_{K}
$$

where

$$
\hat{H}_{K}=\sum_{\mathbf{k} \sigma} \epsilon_{k} c_{\mathbf{k} \sigma}^{\dagger} c_{\mathbf{k} \sigma}+2 J_{K} \sum_{i} \hat{\mathbf{s}}_{c}(i) \cdot \hat{\mathbf{S}}_{i}
$$

describes the conduction sea and its coupling to the localized moments. Here $\hat{\mathbf{s}}_{c}(i) \equiv \frac{1}{2}\left(c_{i \alpha}^{\dagger} \boldsymbol{\sigma}_{\alpha \beta} c_{i \beta}\right)$ describes the spin density of conduction electrons at site $\mathbf{R}_{i}$ through electron creation operators $c_{i \sigma}^{\dagger}=\sum_{\mathbf{k}} c_{\mathbf{k} \sigma}^{\dagger} e^{i \mathbf{k} \cdot \mathbf{R}_{i}}$ and $\boldsymbol{\sigma}_{\alpha \beta}$ are the Pauli matrices.

If a magnetically ordered phase exists, the size of the ordered (condensed) moment can be expressed through the identity:

$$
M_{0}^{2}=\left\langle\left(\hat{\mathbf{S}}_{f}+\hat{\mathbf{s}}_{c}\right)^{2}\right\rangle-3 \int \frac{\mathrm{d}^{d} q}{(2 \pi)^{d}} \int_{0}^{\infty} \frac{\mathrm{d} \omega}{\pi}\left(\frac{1}{2}+\mathrm{n}(\omega)\right) \chi_{\text {tot }}^{\prime \prime}(\mathbf{q}, \omega),
$$

where $n(\omega)$ is the Bose distribution function, and $\chi_{\text {tot }}^{\prime \prime}$ is the dynamical susceptibility of the magnetization

$$
\chi_{t o t}^{\prime \prime}(q) \delta_{a b}=\frac{1}{2} \int \mathrm{d}^{4} x\left\langle\left[\hat{M}_{a}(x), \hat{M}_{b}(0)\right]\right\rangle e^{-i q x}, \quad(q \equiv(\mathbf{q}, \omega), q x \equiv \mathbf{q} \cdot \mathbf{x}-\omega t) .
$$

Here $\hat{\mathbf{M}}(x)=\hat{\mathbf{s}}_{c}+\hat{\mathbf{S}}_{f}$ is the total magnetization. The dynamical susceptibilty can be expanded in terms of both localized and conduction electron contributions $\chi_{\text {tot }}^{\prime \prime}=\chi_{c c}^{\prime \prime}+\chi_{f f}^{\prime \prime}+2 \chi_{f c}^{\prime \prime}$.

Equation (5) offers insight into two alternative ways in which the size of the ordered moment may be reduced:

- Spin zero-point motion, encoded in the second term on the r.h.s. of Eq. (5) which reduces the magnitude of the mean-field ordered moment by transfering spin spectral weight from the ordered moment into the finite frequency part of the spin fluctuations. In a pure insulating antiferromagnet, the $f$-electron ordered moment will be, generally, smaller than the classical value $S$, as follows from Eq. (5):

$$
M_{f}^{2}=S(S+1)-3 \int \frac{\mathrm{d}^{d} q}{(2 \pi)^{d}} \int_{0}^{\infty} \frac{\mathrm{d} \omega}{\pi}\left(\frac{1}{2}+\mathrm{n}(\omega)\right) \chi_{f f}^{\prime \prime}(\mathbf{q}, \omega),
$$

Geometric frustration enhances zero-point motion of spins, transfering spectral weight from the spin-condensate into the fluctuations described by $\chi_{f f}^{\prime \prime}(\mathbf{q}, \omega)$. This reduces the size of the ordered moment, and beyond a critical value of frustration $Q_{c}$, no long-range spin order 
can survive. Examples include the kagomé lattice or square lattice with particular ratio of next-nearest neighbour interaction $J_{2} / J_{1}=1 / 2$. In all these cases, the zero-point motion is concentrated in a region of momentum space surrounding the classical ordering wave-vector Q.

- Kondo screening. This effect can be simply understood as reduction in the first term on the r.h.s. of Eq. (5), due to the antiferromagnetic interaction between the localized electron moment $\mathbf{S}_{f}$ and the conduction electron spin $\mathbf{s}_{c}$. Note that the integral in the last term in Eq. (5) will also decrease, because of the negative contribution of hybridization term $\chi_{c f}$ into the total susceptibility ([6) . It is the mixed component of the susceptibility that, at temperatures below the Kondo temperature $T_{K}$, encodes the Kondo screening, leading to the reduction of the localized moment $S_{f} \rightarrow S-1 / 2$ in the ground state. The important difference from the zero-point motion discussed above, is that $\chi_{c f}^{\prime \prime}(\mathbf{q}, \omega)$ is a local quantity and unlike the effects of spin zero-point motion, the effects of Kondo screening are diffusely distributed in momentum space.

In fact, both zero-point fluctuation and spin-screening effects must be present in a heavy fermion compound, providing the basis for two independent axes in the generalized $Q K$ heavy fermion phase diagram, shown in Fig. 1. The $x$-axis describes the Kondo screening, with a tuning parameter given by the ratio $K=T_{K} / J^{H}$ of the Kondo temperature $T_{K}$ to the characteristic antiferromagnetic coupling strength $J^{H}$. The $y$-axis describes the effect of spin zero-point motion, tuned by the frustration parameter $Q$. We now discuss this diagram in detail, with reference to various concrete model examples.

\section{B. General considerations}

We now turn to a more detailed discussion of the "QK" diagram, combining Kondo screening $(K)$ and frustration $(Q)$ as the $x$ and $y$-axes. Let us first discuss the axes of this diagram. In his semi-qualitative phase diagram, Doniach $\underline{1}$ argued that the transition from magnetically ordered phase into a paramagnetic heavy Fermi liquid occurs as a function of increasing Kondo coupling $J_{K}$ at a point where the Kondo temperature becomes comparable with the strength of the $R K K Y$ interaction $K_{c}=T_{K} / J^{H} \sim 1$. In a realistic model the RKKY scale responsible for long range magnetic order scales as $J^{H} \sim J_{K}^{2}$, while the Kondo scale is exponential in $J_{K}$ and eventually wins as the latter increases, $T_{K} \sim D \exp \left(-D / 2 J_{K}\right)$ (where $D$ is the conduction electron bandwidth). 
An equivalent way to think of Doniach's phase diagram is to assume that the RKKY strength and the Kondo temperature are independent quantities, while their ratio $K=T_{K} / J^{H}$ is the relevant parameter, as plotted on the horizontal axis in Fig. 1.

The $y$-axis is the frustration axis. In real systems, the strength of zero-point motion $Q$ can be associated with frustration, associating it for example, with the ratio of competing bond strengths. From a theoretical stand-point, the enhancement of zero-point spin fluctuations can also be accomplished by considering a tuning of the spin $S$ of the local moments. In practice this is done in a large- $S$ or a large- $N$ expansion using a Schwinger boson description of the local moments. In these more theoretical approaches, we can loosely identify $Q \sim N /(2 S)$ where $N$ is the number of components of a $\mathrm{SU}(N)$ or $\mathrm{Sp}(N)$ spin. There is a small caveat associated with these approaches, for once the size of the Kondo spin becomes greater than $S=1 / 2$, we require $2 S$ screening channels to fully screen the local moment when the Kondo coupling is turned on, requiring that a large- $N$ or large- $S$ expansion be done with a Kondo lattice in which there are then $k=2 S$ conduction bands, each individually screening the local moments $26,27$.

In the absence of coupling to conduction electrons (i.e in insulating magnets), the effect of quantum frustration is relatively well understood. For example, in spin-wave theory, the magnetic order disappears above some critical value $Q_{c}=1 / S_{c}$ (on a square lattice $S_{c} \sim 0.2$ ) $\underline{22}$. At higher values of $Q$, a dimerized valence bond solid (VBS) phase, or alternatively spin liquid - a state with no broken symmetry, are expected to develop.

Once we combine the Kondo $(K)$ and frustration $(Q)$ effects into a single diagram, antiferromagnetism occupies the small $K$, small $Q$ corner of the phase diagram. Since there is only one antiferromagnetic phase, there must be a single phase boundary that connects the quantum phase transitions at $K=K_{c}$ on the x-axis and $Q=Q_{c}$ on the y-axis.

The paramagnetic phases that exist outside the AFM phase boundary differ qualitatively at small and large $K$. At large $K$, the quenching of the localized moments in the Kondo lattice liberates spin into the conduction sea, and we expect a Fermi surface volume $V_{F S}$ determined by

$$
\frac{V_{F S}}{(2 \pi)^{D}}=n_{e}+\frac{n_{s}}{2}
$$

where $n_{e}$ is the density of electrons per unit cell per spin index while $n_{s}$ is the number of spins per unit cell. This expresses the fact that each spin liberates one heavy electron degree of freedom, or half an electron per spin index. Now at small finite $K$, the Kondo effect will not take place so that $V_{F S} /(2 \pi)^{D}=n_{e}$, implying that

$$
\frac{\Delta V_{F S}}{(2 \pi)^{D}}=\frac{n_{s}}{2}
$$


between small and large $K$ phases. Now, the volume of the Fermi surface is only defined modulo $(2 \pi)^{D}$, so an increase in the electron count per unit by $n_{S} / 2$ will lead to a change in the Fermi surface volume only if $n_{S}$ is an odd number. In this case, provided no symmetries are broken, the large and small $K$ Fermi surfaces cannot be connected continuously, and must be separated by a (zero temperature) quantum phase transition. Of the five well-known lattices, the square, triangular and kagomé lattices have an odd number of spins per unit cell and are expected to exhibit the small-to-large Fermi surface transition. By contrast, the Shastry-Sutherland and pyrochlore lattices have four spins per unit cell and thus cannot be guaranteed to exhibit a phase transition between small and large $K$ paramagnetic phases:

\begin{tabular}{l|c|c} 
Lattice & Spins/u. cell & spin-liquid-FL QCP \\
\hline Square/Triangular & 1 & $\checkmark$ \\
Kagome & 3 & $\checkmark$ \\
Shastry-Sutherland & 4 & $\mathrm{x}$ \\
Pyrocholore & 4 & $\mathrm{x}$
\end{tabular}

Most studies of the frustrated square lattice Heisenberg model suggest that rather than a spin liquid, a valence bond solid forms in the presence of strong frustration, breaking the lattice symmetry. In this case, the phase boundary to the heavy Fermi liquid will extend to finite temperature. The important point however, is that under quite general conditions, provided there is an odd number of spins per unit cell, in the absence of antiferromagnetic order, there must exist some kind of quantum phase transition between the small- and large- Fermi surface state.

This is of course, a minimal requirement, which does not preclude more complex transitions between the small and large Fermi surface states. As we shall see, this may even be possible in those cases with an even number of spins per unit cell. To address these questions requires a more microscopic approach to the Hamiltonian. The important point is that from the $Q K$ diagram, we are able to deduce that in a Kondo lattice with an odd number of spins per unit cell, the "delocalization" line for the $f$-electrons must separate from the antiferromagnetic phase transition at large enough frustration parameter $Q$. This has two qualitative consequences:

- Kondo stabilization of the "spin liquid". The qualitative form of the AFM phase boundary in the $Q K$ diagram raises the fascinating possibility that once $K>0$, Kondo screening reduces the size of the local moment, reducing the critical value $Q_{c}$ for the formation of a spin liquid. This "Kondo stabilization" of the spin liquid state was first suggested by Coleman and Andrei in their 1987 adaptation of the RVB pairing idea to heavy fermions. $\underline{28}$. 
- Local moment-spin-density wave transition. It is very natural to assume that when the $f$-electron delocalization line meets the magnetic phase boundary, it continues on inside the magnetic phase. The appeal of this conjecture $\frac{14}{4}$, is that it permits a separation of the AFM phase into "localized" and a "spin-density wave" (SDW) regions, allowing for the possibility of heavy fermion materials in which the quantum critical point is described by an itinerant, Hertz-Millis scenario $\underline{29}, 30$. This boundary may take the form of a Lifschitz transition in the underlying Fermi surfaces.

Beyond these general considerations, very little is known about the detailed form of the global phase diagram, and a number of variant forms have been proposed (Fig. 4), largely motivated by experiment. One possibility, motivated largely by experiment, is that the antiferromagnetic phase boundary and the spin delocalization line merge over finite region of the phase diagram ${ }^{31}$ as illustrated in Fig. 4(a), allowing for the possibility of a region of the phase boundary governed by a "local quantum critical point" where the antiferromagnetism and the localization of the $f$-electrons occurs simultaneously. A second possibility, also motivated by experiment, is that the transition between small and large Fermi surface metal takes place via an intermediate "strange metal" phase depicted in Fig. 4(b). We shall later return to discuss these scenarios in the context of experimental observations.

a)

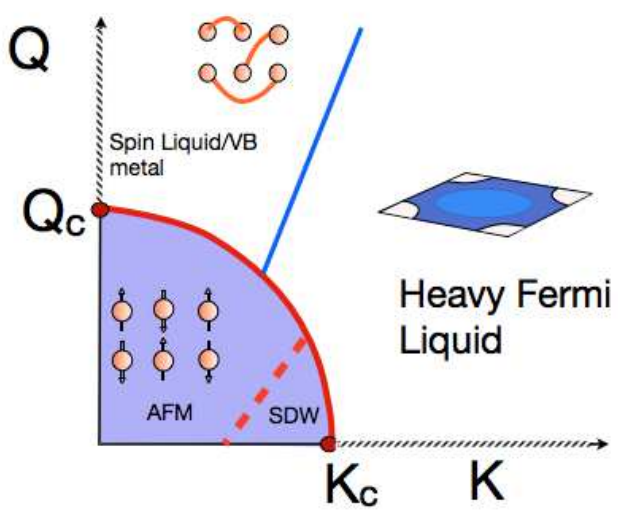

b)

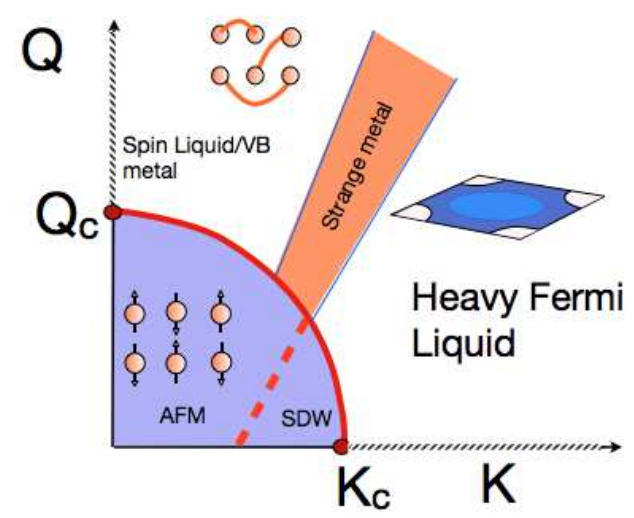

Figure 4: (Colour online) Two alternative scenarios for the global $Q K$ phase diagram: (a) in which the $f$ electron delocalization line merges with the antiferromagnetic boundary over a finite region ${ }^{31}$, (b) in which the transition between small and large Fermi surface takes place via an intermediate "strange metal" phase. 


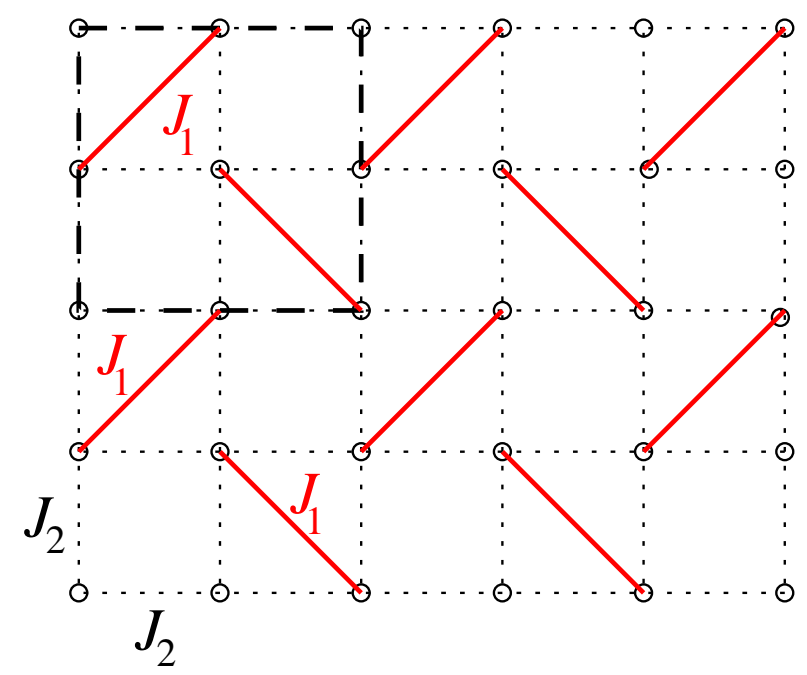

Figure 5: (Colour online) Shastry-Sutherland lattice with each vertex occupied by a localized electron spin. The next nearest neighbours interact via Heisenberg interaction $J_{1}$ along alternate diagonals, favouring the dimerized ground state, while the nearest neighbour interaction $J_{2}$ favours the Néel antiferromagnet. The $2 \times 2$ elementary unit cell is shown with dashed line, corresponding to the reduced Brillouin zone in $\mathrm{k}$-space.

\section{The Shastry-Sutherland Kondo lattice}

Here we discuss a particularly simple example of a frustrated Kondo model: the "ShastrySutherland" Kondo lattice. This lattice is obtained by Kondo-coupling conduction electrons on a square lattice to a frustrated Shastry-Sutherland (SS) spin model 24 . This latter is a checker-board lattice of Heisenberg $S=1 / 2$ moments, in which the coloured squares of the checker board contain alternating diagonal bonds of strength $J_{1}$, as shown in Fig. 5. Each vertex of the SS lattice is thus connected to one partner on the diagonal by $J_{1}$ (thick solid lines) and to 4 nearest neighbours by $J_{2}$ (thin dotted lines). The frustration parameter is the ratio $J_{1} / J_{2}$ of the diagonal to the nearest-neighbour antiferromagnetic couplings $J_{2}$. Recently, an experimental realization has been found in a quasi-two-dimensional heavy fermion system $20 \mathrm{Yb}_{2} \mathrm{Pt}_{2} \mathrm{~Pb}$, with $\mathrm{Yb}^{3+}$ ions forming the Shastry-Sutherland lattice, and in its Ce analogs 32 .

From the theoretical stand-point, the Shastry-Sutherland lattice has a clear advantage that the spin-ground-state at large frustration is a valence-bond solid with spin-singlets arranged on the diagonal bonds and a well-defined wave-function. Unlike other "spin liquid" ground-states, this dimer state was proven to be the exact ground $\operatorname{state}^{24}$ of the SS model (provided $J_{1} \gtrsim 2 J_{2}$ ) and in particular, is well captured by large- $N$ expansion. It is to our knowledge, the only frustrated Kondo lattice where the large- $N$ expansion can reliably examine the effect of Kondo coupling. This 
is therefore an ideal starting point to examine the combined effects of the Kondo screening and strong frustration.

The Hamiltonian of the Shastry-Sutherland Kondo lattice model is

$$
\hat{H}_{S S K}=\hat{H}_{K}+\hat{H}_{S S},
$$

where

$$
\begin{aligned}
\hat{H}_{K} & =\sum_{\mathbf{k} \sigma} \epsilon_{k} c_{\mathbf{k} \sigma}^{\dagger} c_{\mathbf{k} \sigma}+2 J_{K} \sum_{i} \hat{\mathbf{s}}_{c}(i) \cdot \hat{\mathbf{S}}_{i} \\
\hat{H}_{S S} & =J_{1} \sum_{\square, \square} \mathbf{S}_{k} \cdot \mathbf{S}_{l}+J_{2} \sum_{\langle i, j\rangle} \mathbf{S}_{i} \cdot \mathbf{S}_{j}
\end{aligned}
$$

describe the Kondo and magnetic parts of the Hamiltonian. Here $\square, \nabla$ refers to the sum over plaquettes with alternating diagonal bonds. The dispersion of the conduction sea is determined by a tight binding model on a regular square lattice, with hopping of strength $t$ between nearest neighbour sites, so that $\epsilon_{\mathbf{k}}=-2 t\left(\cos k_{x}+\cos k_{y}\right)-\mu$. In the SS lattice, the ratio of the diagonal to the nearest neighbour interaction, $Q=J_{1} / J_{2}$, plays the role of the frustration parameter.

In the absence of Kondo screening, $J_{K}=0$, Shastry and Sutherland 24 showed that the frustrated lattice has a dimerized ground state at large $Q$,

$$
\left|\Psi_{\mathrm{SS}}\right\rangle=\prod_{\square, \square}|d\rangle_{k l}
$$

where $|d\rangle_{k l}=\left(\left|\uparrow_{k} \downarrow_{l}\right\rangle-\left|\downarrow_{k} \uparrow_{l}\right\rangle\right) / \sqrt{2}$ defines a dimer (singlet) on the diagonal bond. In the opposite limit, $Q \ll 1$, the ground-state is a Néel AFM. The transition between the two phases is still controversial. High-temperature series expansion ${ }^{33}$, exact diagonalization ${ }^{34}$ and variational studies 35 point to a direct transition between the dimer phase and the Néel ground state at $Q_{c}=\left(J_{1} / J_{2}\right)_{c}=1.43 \pm 0.02$. On the other hand, a number of studies ${ }^{36}-39$ suggest that an intermediate phase exists between $1.1 \lesssim Q \lesssim 1.65$ with different proposals as to its nature, including a helical AFM, a plaquette singlet phase or a columnar phase.

The SS lattice has four spins per unit cell and following the counting argument of the last section, a priori we might expect a continuous evolution from the the small Fermi surface metal to large Fermi surface heavy electron state.

However, there is a limit in which a phase transition does occur: the half-filled lattice. With one conduction electron per site, the $K=0$ state is a metal, whereas the $K=\infty$ state is a Kondo insulator, implying a metal-insulator transition at half filling which separates the small and large $K$ limits. Since there is no change in symmetry, this metal-insulator transition will be a zero temperature quantum critical point, with gapless charge excitations. 
When we dope away from half filling, we expect the quantum critical point to influence the physics at finite doping, giving rise to instabilities. Indeed, as we now argue, based on both strong coupling arguments and the large $N$ expansion, at low enough temperatures the large and small Fermi surface phases are likely to be unstable to a $d_{x y}$-wave superconducting instability induced by the magnetic coupling along the diagonal bonds. To this end, we will limit our discussion to the limiting case of infinite frustration where $J_{2}=0(Q=\infty)$.

Consider first the strong-coupling limit of the Shastry-Sutherland Kondo lattice, in which both the Heisenberg and Kondo couplings are much larger than the hopping, $J_{1}, J_{K} \gg t$. We can consider two extreme limits:

(i) Weak Kondo coupling, $J_{1} \gg J_{K} \gg t$. It is useful to visualize the situation using an RVB notation to describe the configuration of the singlet bonds between the electrons and the localized moments. Single electrons will have their energy lowered by an amount of order $-J_{K}^{2} / J_{1}$ by inducing a virtual resonances of the dimer bond in which one end of the bond re-attaches to the conduction electron, forming a Kondo singlet. This effect can only occur with a singlyoccupied conduction electron site, and will be absent with two electrons above the same site, thereby generating an effective repulsive on-site $U \sim J_{K}^{2} / J_{1}$ between electrons. By contrast, when two electrons 'hover' above a diagonal valence bond, that valence bond can resonate into the conduction sea. In the second order perturbation theory, this leads to an induced antiferromagnetic interaction between conduction electrons of strength $g \sim J_{K}^{2} / J_{1}$ across the alternate diagonals of the electron lattice, so that the effective model describing the electrons at small $K$ is given by

$$
H_{e f f}=\sum_{\mathbf{k} \sigma} \epsilon_{k} c_{\mathbf{k} \sigma}^{\dagger} c_{\mathbf{k} \sigma}+U \sum_{j} n_{j \uparrow} n_{j \downarrow}+g \sum_{\square, \square} \boldsymbol{\sigma}_{k} \cdot \boldsymbol{\sigma}_{l}
$$

where $g, U \sim J_{K}^{2} / J_{1}$. The weak antiferromagnetic interaction between the electrons across the diagonals of the alternate plaquettes will couple to the divergent Cooper pair instability in the $d_{x y}$ channel, giving rise to a Cooper instability into a weakly paired state with $d_{x y} \sim \sin k_{x} \cdot \sin k_{y}$ symmetry (note that repulsive $U$ will suppress the competing instability into an extended s-wave state). In general all other competing instabilities will not have a divergent susceptibility, and will be absent for small enough $J_{K} / J_{1}$.

(ii) Now consider the large $K$ limit where $J_{K} \gg J_{1} \gg t$. If we have exactly one electron per site, the state formed is a Kondo insulator, with Kondo singlets at each site (Fig. 6 (a)):

$$
|\mathrm{KI}\rangle=\prod_{i} \frac{1}{\sqrt{2}}\left(c_{i \uparrow}^{\dagger} f_{i \downarrow}^{\dagger}-c_{i \downarrow}^{\dagger} f_{i \uparrow}^{\dagger}\right)|0\rangle
$$


(a)

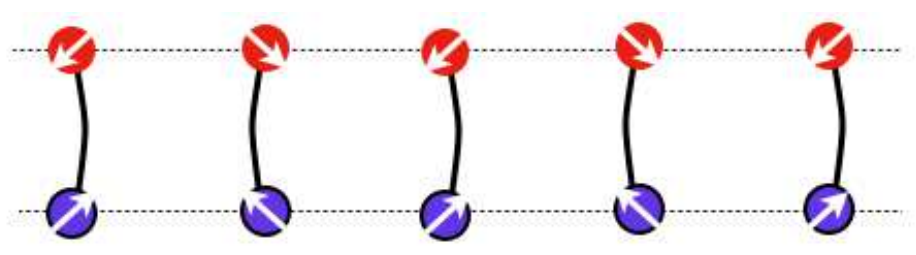

(b)

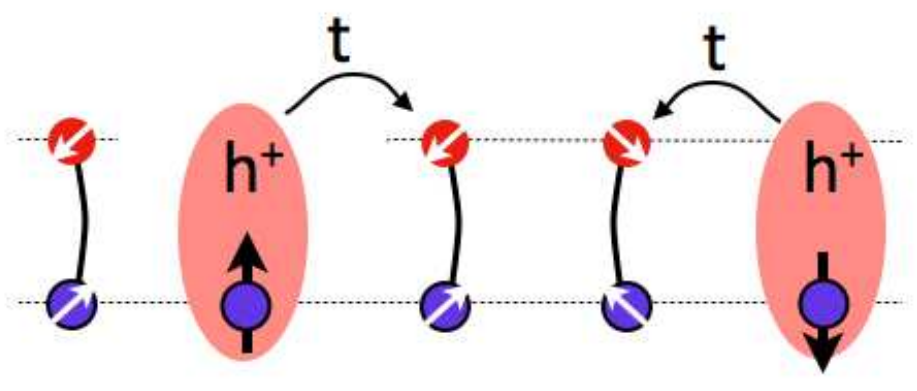

(c)

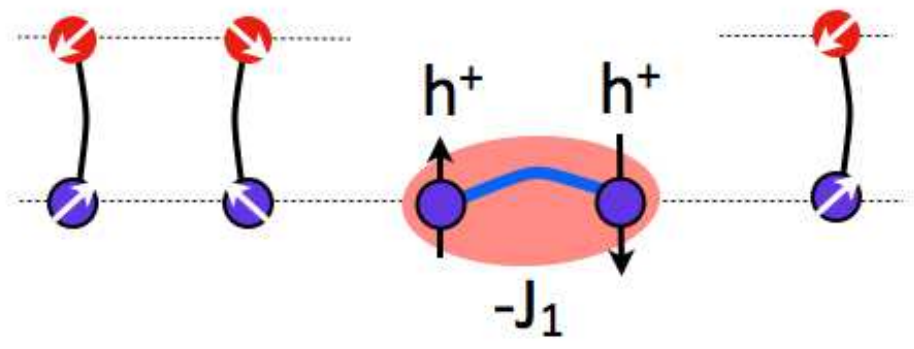

Figure 6: (Colour online) (a) In the strong coupling limit $J_{K} \gg J_{1} \gg t$, the ground state of the half-filled electron lattice is a Kondo insulator, with a singlet formed at each site between each localized moment (blue) and conduction electron (red), see Eq. (14). (b) When electrons are removed from the Kondo-insulator, the resulting positively charged holes carry the spin of the underlying local moment and can hop from site to site, forming a heavy fermion metal. (c) When two holes are created above the diagonal sites A and B of the underlying Shastry-Sutherland lattice, they form a singlet with binding energy $-J_{1}$.

By removing electrons from this state, one creates the "holes" (Fig. 6(b)) that form the excitations of the large Fermi surface state, $\tilde{h}_{j \sigma}^{\dagger} \equiv-c_{j-\sigma} \operatorname{sgn}(\sigma)$, so that

$$
\tilde{h}_{j \sigma}^{\dagger}|\mathrm{KI}\rangle=\frac{1}{\sqrt{2}} f_{j \sigma}^{\dagger} \prod_{i \neq j} \frac{1}{\sqrt{2}}\left(c_{i \uparrow}^{\dagger} f_{i \downarrow}^{\dagger}-c_{i \downarrow}^{\dagger} f_{i \uparrow}^{\dagger}\right)|0\rangle .
$$

Creating a hole in the half-filled lattice (i.e. an empty site) destroys the Kondo singlet and thus has an energy cost $J_{K}$ which can be absorbed into the chemical potential, $\tilde{\mu}=\mu-J_{K}$. The holes can hop from site to site, as illustrated in Fig. 6(b). While generically, there are no spin dimers 
present, if two holes happen to come together across the diagonal of a plaquette, then a dimer can form on the underlying Shastry-Sutherland $f$-spin lattice, as shown schematically in Fig. 6(x). The energy of such configuration will be lowered by an amount of order $-J_{1}$ in the first order of the perturbation theory. In this way, the effective Hamiltonian at large $K$ will take the form

$$
H_{e f f}^{\prime}=\sum_{\mathbf{k} \sigma} \tilde{\epsilon}_{k} \tilde{h}_{\mathbf{k} \sigma}^{\dagger} \tilde{h}_{\mathbf{k} \sigma}+J_{1} \sum_{\square, \square} \tilde{\boldsymbol{\sigma}}_{k} \cdot \tilde{\boldsymbol{\sigma}}_{l}
$$

where $\tilde{h}_{\mathbf{k} \sigma}^{\dagger}$ creates a heavy $f$-hole, as per Eq. (15), with dispersion $\tilde{\epsilon}_{\mathbf{k}}=+t\left(\cos k_{x}+\cos k_{y}\right)-\tilde{\mu}$ and spin $\tilde{\boldsymbol{\sigma}}_{i}=\frac{1}{2}\left(\tilde{h}_{i \alpha}^{\dagger} \boldsymbol{\sigma}_{\alpha \beta} \tilde{h}_{i \beta}\right)$. Because of the effective antiferromagnetic coupling on the alternating diagonals, this "large Fermi surface" state will also be susceptible to the $d_{x y}$ superconducting instability.

In this way, strong coupling arguments suggest the prevalence of a $d_{x y}$ superconductor at both large and small values of $K$. Adiabaticity can then be used to argue that unless anything unforeseen occurs, the two superconducting states are connected across the phase diagram, shown schematically in Fig. 7 .

A similar set of arguments can be advanced using a large $N$ treatment of the Shastry-Sutherland Kondo lattice. Let us again consider the limit of infinite frustration $J_{2}=0(Q \rightarrow \infty)$ when the dimer phase is stable. The dimer phase can be captured by using the fermionic representation of spins, $\mathbf{S}_{i}=\frac{1}{2} f_{i \alpha}^{\dagger} \boldsymbol{\sigma}_{\alpha \beta} f_{i \beta}$, subject to the occupancy constraint $n_{f}=1$. We introduce an anomalous operator

$$
B_{i j}^{\dagger}=\sum_{\sigma} \operatorname{sgn}(\sigma) f_{i \sigma}^{\dagger} f_{j-\sigma}^{\dagger}
$$

which creates a singlet pair of $f$-electrons on the diagonal links of the Shastry-Sutherland model. While physical spins are described by $\mathrm{SU}(2)$ group, the above expression can be easily generalized to the spins that belong to symplectic $\operatorname{Sp}(N)$ group, with $\sigma=-\frac{N}{2}, \ldots \frac{N}{2}$. In the large- $N$ mean field theory, the dimer phase is described by the non-zero expectation value $\Delta=J_{1}\left\langle B_{i j}\right\rangle / N$, and the spin Hamiltonian can be written as

$$
\tilde{H}_{S S}=-\sum_{i}\left(\Delta_{\square} B_{i j}^{\dagger}+\text { h.c. }\right)-\sum_{k}\left(\Delta_{\square} B_{k l}^{\dagger}+\text { h.c. }\right)+N \frac{\left|\Delta_{\square}\right|^{2}}{J_{1}}+N \frac{\left|\Delta_{\square}\right|^{2}}{J_{1}},
$$

where by symmetry one expects the dimer averages to be the same on the alternate diagonals, up to an arbitrary phase: $\Delta_{\square}=\Delta_{\square} e^{i \phi}$.

The heavy Fermi liquid ground state, on the other hand, can be characterized by non-zero on-site expectation value of hybridization $V_{i}=-J_{K} \sum_{\sigma}\left\langle c_{i \sigma}^{\dagger} f_{i \sigma}\right\rangle / N$. The full large- $N$ mean-field 


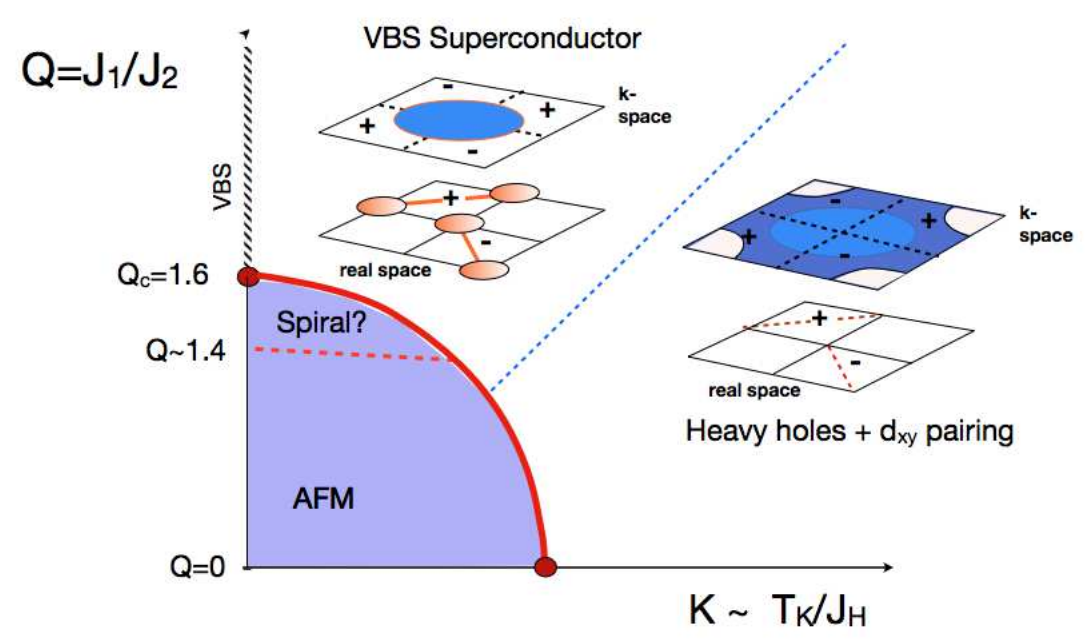

Figure 7: (Colour online) Schematic phase diagram of the Shastry-Sutherland-Kondo model. The frustration axis denotes $Q=J_{1} / J_{2}$. At half-filling, there is a metal insulator transition from the insulating dimer phase on the left into a $d$-wave superconductor with a large Fermi surface on the right. At a generic filling, both phases are metallic and the transition becomes a crossover.

Hamiltonian then becomes:

$$
H^{M F}=\sum_{\mathbf{k} \sigma} \epsilon_{k} c_{\mathbf{k} \sigma}^{\dagger} c_{\mathbf{k} \sigma}+\tilde{H}_{S S}+\sum_{i \sigma}\left(V f_{i \sigma}^{\dagger} c_{i \sigma}+\text { h.c. }\right)+N \frac{|V|^{2}}{J_{K}} .
$$

In the limit $K \ll 1$, the dimer phase dominates, with non-zero expectation value of $\Delta_{\square}$ and $\Delta_{\square}$. In the opposite limit of strong Kondo interaction, $V$ will acquire a non-zero expectation value. Generically, both order parameters may co-exist in a certain region of the phase diagram.

It is easy to show that such coexistence describes a superconducting instability of the conduction electrons within this large- $N$ mean field theory. This is related to the fact that the product of the two mean-field order parameters $V_{i} V_{j} B_{i j}$ translates into the average:

$$
V_{i} V_{j} \Delta_{i j} \propto\left\langle\left(c_{i}^{\dagger} f_{i}\right) \cdot \hat{B}_{i j}^{\dagger} \cdot\left(c_{j}^{\dagger} f_{j}\right)\right\rangle \propto\left\langle c_{i \sigma}^{\dagger} c_{j-\sigma}^{\dagger} \operatorname{sgn}(\sigma)\right\rangle,
$$

i.e. it describes pairing of conduction electrons on the diagonals of the Shastry-Sutherland lattice. It is clear from the above strong coupling arguments that the extended $s$-wave pairing will be at disadvantage compares with the $d$-wave pairing, and so the above anomalous average will describe $d_{x y}$ pairing.

The resulting phase diagram of the Shastry-Sutherland Kondo lattice model is shown schematically in Fig. 7. Current experimental realization of the Shastry-Sutherland Kondo lattice, including 
$\mathrm{Yb}_{2} \mathrm{Pt}_{2} \mathrm{~Pb}$ compound $\underline{20}$ and its cerium analogs 32 , lie either in the antiferromagnetic region, or on the edge of the paramagnetic/spin liquid region of the phase diagram. The possibility of a superconducting ground-state, co-existing with dimer order is an fascinating possibility for future work. More studies of the Shastry-Sutherland Kondo model are needed, and its full understanding, through theory and experiment, appears to be an important step towards future understanding of the interplay of frustration and Kondo effects in heavy fermion systems.

\section{EXPERIMENTAL CONSIDERATIONS}

(a)

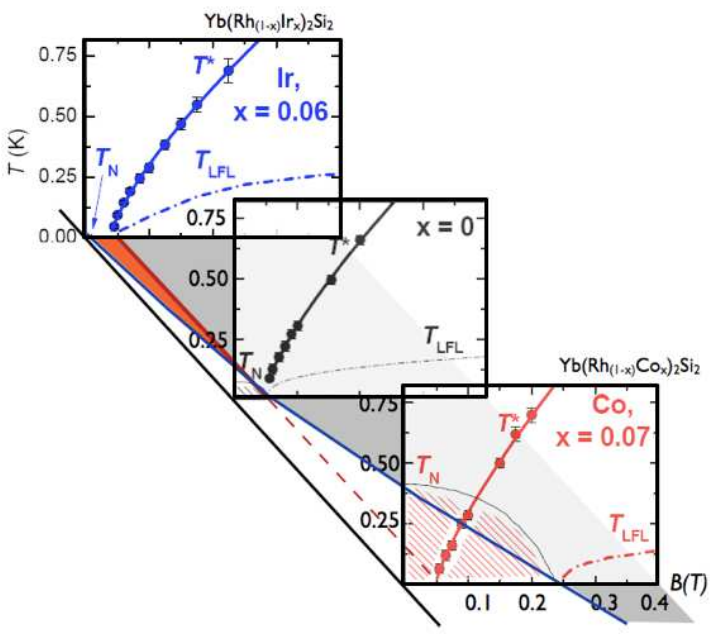

(b)

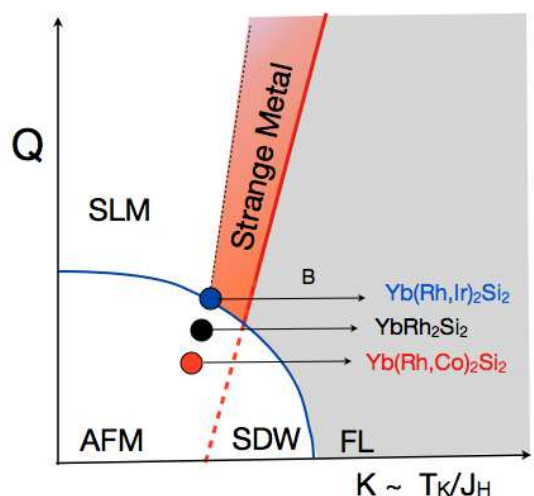

Figure 8: (a) Temperature-field phase diagrams for Co- and Ir-doped $\mathrm{YbRh}_{2} \mathrm{Si}_{2}$ after Ref. [5, superposed onto the $Q K$ diagram. (b) Interpretation of the experiments ${ }^{\underline{5}}$ on doped $\mathrm{YbRh}_{2} \mathrm{Si}_{2}$ within the $Q K$ diagram, the arrows showing the effect of applying magnetic field.

There is mounting experimental evidence for the importance of magnetic frustration in heavy fermion physics. While the examples of $f$-electron metals on strongly geometrically frustrated lattices are very rare, such as e.g. the pyrochlore compound $\operatorname{Pr}_{2} \operatorname{Ir}_{2} \mathrm{O}_{7}$, there are indications that a number of compounds crystallizing in an a priori non-frustrated tetragonal lattice, become de facto frustrated thanks to competing RKKY interactions between the nearest and next nearest neighbours. The existence of a spiral antiferromagnetic phase ${ }^{40}$ in $\mathrm{CeRhIn}_{5}$ is a particularly striking example of such a competition, on a par with apparent absence of any long-range magnetic order 
in the sister superconducting compound $\mathrm{CeCoIn}_{5}$ (although the Néel order can be induced by doping with $\left.\mathrm{Cd}^{41}\right)$. In another well-studied heavy fermion material, $\mathrm{YbRh}_{2} \mathrm{Si}_{2}$, the very tiny Néel temperature $T_{N} \approx 70 \mathrm{mK}^{42}$, which can be further suppressed by doping with $\mathrm{Ir}^{\underline{5}}$, also points to the importance of magnetic frustrations in this system.

It is in these tetragonal lattice systems that the small- to large- Fermi surface transition has been most clearly established. There is strong experimental evidence from the Hall effect measurements that applying magnetic field to pure $\frac{43}{\underline{3}}$ and $\operatorname{doped}^{\underline{5}} \mathrm{YbRh}_{2} \mathrm{Si}_{2}$ leads to a jump in the Fermi surface volume. A similar phenomenon occurs when hydrostatic pressure is applied to $\mathrm{CeRhIn}_{5}$, when a clear change of the Fermi surface topology is seen in the de Haas-van Alphen measurements in the field-induced normal state $\stackrel{44}{ }$. As we argued above, our $Q K$ phase diagram provides reason to believe that the small-to-large Fermi surface transition should be present also in the spin-liquid phase, independent of the antiferromagnetic phase boundary. This point-of-view is also supported by other theories $\underline{26}, 45$.

Indeed, recent measurements on doped $\frac{5}{5}$ and pressurized ${ }^{\underline{4}} \mathrm{YbRh}_{2} \mathrm{Si}_{2}$ unambiguously show that the small-to-large Fermi surface transition is separate from the conventional antiferromagnetic quantum critical point. This is illustrated in Fig. 8, in which we attempted to put the existing data on Co- and Ir-doped $\mathrm{YbRh}_{2} \mathrm{Si}_{2}$ onto the theoretical $Q K$ diagram. It has to be kept in mind that generically, changing experimental parameters, such as pressure, doping or magnetic field, may involve simultaneous changes along both the Doniach and frustration axes in the $Q K$ phase diagram.

In the case of doped $\mathrm{YbRh}_{2} \mathrm{Si}_{2}$, the data suggest $\mathrm{t}^{-5}$ that applying magnetic field drives the system along the horizontal $K$-axis, as shown with arrows in schematic phase diagram in Fig. $8 \mathrm{~b}$ ). Crucially, the Co-doped material exhibits first the jump in the Hall coefficient at smaller field, and then an AFM to paramagnet transition, which suggests that it is likely to be deeper inside the AFM phase then pure $\mathrm{YbRh}_{2} \mathrm{Si}_{2}$, as shown by the red circle in Fig. $8(\mathrm{~b})$. By contrast, the Ir-doped material first undergoes a transition into a paramagnet, and then develops a large Fermi surface characteristic of a heavy Fermi liquid. The strange phase that lies in between cannot thus be a conventional Fermi liquid, and on the schematic phase diagram Fig. 8(b) we denote it as a "strange metal" phase, forming a wedge along the line of small- to large- Fermi surface transition. It is tempting therefore to place the Ir-doped $\mathrm{YbRh}_{2} \mathrm{Si}_{2}$ closer to the spin-liquid phase (blue circle in Fig. 8b) then the pure compound (black circle).

Intriguingly, the "strange metal" phase on the $Q K$ phase diagram may actually be realized in the recently discovered $\beta-\mathrm{YbAlB}_{4}$ compound ${ }^{8,9}$. This material appears to be quantum critical 
without any external tuning, as characterized by anomalous critical exponents and $T / B$ scaling. Unless nature has fortuitously placed $\beta-\mathrm{YbAlB}_{4}$ right at a quantum critical point, this material is most probably an example of "strange metal" phase, shown as the coloured wedge in Fig. 8(b).

Furthermore, the "quantum critical phase" in $\beta-\mathrm{YbAlB}_{4}$ appears to be unstable to application of magnetic field. A tiny field, comparable to the Earth's magnetic field, is sufficient to drive the material back into a Fermi liquid. That magnetic field is a relevant perturbation to this critical phase is a very important observation which deserves further experimental and theoretical work. It is worth noting that structurally very similar compound, $\alpha-\mathrm{YbAlB}_{4}$, shares the same features as the $\beta$-phase in its thermodynamic properties (susceptibility, specific heat) $\frac{9}{}$ above $T^{*} \sim 2 \mathrm{~K}$, yet has a Fermi liquid ground state. The possibility that by applying hydrostatic pressure one may be able to drive $\beta-\mathrm{YbAlB}_{4}$ into a Fermi liquid phase, just as $\alpha-\mathrm{YbAlB}_{4}$ is at ambient pressure, is very intriguing and deserved experimental attention.

One of the conclusions arising from theoretical considerations presented earlier, is that the spin liquid state (or valence bond solid, as in the Shastry-Sutherland case) may be reached either by increasing the Doniach ratio $K=T_{K} / J^{H}$ or by increasing the amount of frustration $Q$. In fact it has been recently proposed ${ }^{6}$ that a number of experimental systems, such as YbAgGe and Gedoped $\mathrm{YbRh}_{2} \mathrm{Si}_{2}$ may lie in the vicinity of the point where the three phases cross in the $Q K$ phase diagram in Fig. 8(b). In this case it should be possible, in principle, to drive these systems into a spin liquid state by applying either (chemical) pressure or magnetic field.

\section{CONCLUSIONS AND OUTLOOK}

Our paper has discussed and illustrated how a unified consideration of the effects of spin zeropoint fluctuations (denoted by "Q") and the Kondo screening (encoded by "K") leads to a two dimensional global phase diagram of the Kondo lattice. In particular, general arguments based on the $Q K$ diagram lead us to conclude that the antiferromagnetic phase boundary and the $f$ delocalization transition may be partially, if not totally independent of one another. This possibility was first observed theoretically a number of years ago $\underline{45}$, and if correct, may prove rather liberating for the theoretical community, allowing us to split the problem of quantum criticality into two separate studies of $f$-electron magnetization and localization.

Previously, the various non-Fermi liquid properties observed in the vicinity of the AFM phase transition of heavy fermion compounds, such as the quasi-linear resistivity $\rho \sim \rho_{0}+T^{\alpha}(\alpha \sim 1)$ and the logarithmic temperature dependence of the specific heat $\left(C_{V} / T \sim-\ln T\right)$ were phenomeno- 
logically associated with a single transition. Viewed from the new perspective, it is tempting to associate many of these features with $f$-electron delocalization, rather than the development of magnetism, per se. Senthil $\underline{46}$ has proposed that the small to large Fermi surface transition will involve a critical Fermi surface, reminiscent of that found in Luttinger liquids. It would be fascinating indeed, if such a scenario separated the spin and heavy fermi liquids.

To provide a concrete example, we have considered above the Shastry-Sutherland Kondo (SSK) lattice model, which is believed to be experimentally realized 20 in the heavy fermion compound $\mathrm{Yb}_{2} \mathrm{Pt}_{2} \mathrm{~Pb}$. Its beauty lies in the fact that while being an example of a fully frustrated spin model (provided $Q \gg 1$ ), the ground state of the Shastry-Sutherland model is known exactly. This lifts the uncertainty as regards the ground state, which is otherwise present in virtually all examples of geometrically frustrated spin models. In particular, the SSK model lends itself to large- $N$ treatment. The combination of the latter, together with strong-coupling arguments, allowed us to sketch qualitatively the $Q K$ phase diagram of the model. Ironically, our theory predicts that, with the exception of the special case of half-filling, the quantum critical point is most likely replaced by the $d$-wave superconducting phase which prevails over the entire zero-temperature phase diagram. Nevertheless, this model provides a useful insight into the interplay of frustration and Kondo effect in heavy fermion systems and warrants further detailed studies, both theoretical and experimental.

Historically, the advancement of a theory of classical criticality benefited from a whole host of developments - the solution of the 2D Ising model, the development of classical Monte Carlo methods that opened the physics to numerical analysis, the idea of the renormalization group, the abstraction of phase transitions in terms of a continuum Ginzburg-Landau theory, and lastly the development of new kinds of controlled mathematical treatments, such as the $\epsilon$ and the $1 / N$ expansion.

It would seem likely that a parallel set of developments are required in order for us to understand the nature of quantum criticality in heavy fermion materials. A number of useful directions seem to present themselves:

- Exploration of fully frustrated Kondo lattices. Kondo generalizations of the ShastrySutherland and kagomé lattice may offer the hope of characterizing the $f$-electron delocalization transition without the complications of magnetism. Present fermionic theories $\underline{47}-\underline{49}$ of the Kondo lattice cannot describe the transition into the magnetically ordered state.

- The development of the idea of "local quantum criticality" $\underline{50,51}$ to incorporate soft charge degrees of freedom as a possible approach to $f$-electron delocalization. To date, ideas of 
local quantum criticality have focussed on the notion that the soft critical excitations are the magnetic spins themselves. Yet, when spins of local magnetic moments delocalize, the critical spin excitations must also involve critical charge degrees of freedom. The inclusion of the latter, possibly as zero energy charged fermions, into a locally quantum critical theory may prove fruitful in this respect.

- One of the unsolved problems, is to find a controlled expansion that unifies magnetism with the Kondo effect. The large- $N$ Schwinger boson approach ${ }^{26}$ appears to offer hope in this respect. The Schwinger boson large- $N$ limit of the Kondo lattice involves self-consistent integral equations which have been solved for the one and two impurity Kondo models, but which remain unsolved for the Kondo lattice. The solutions of these integral equations at the $f$-delocalization transition could shed considerable light on the nature of heavy fermion quantum criticality.

- The development of radically new kinds of large $N$ expansion based on an idea from String theory sometimes called "holography" and the "Anti-de Sitter / Conformal Field Theory" (AdS/CFT) correspondence.

We should like to end with a few comments on this last approach. The AdS/CFT correspondence is a conjecture from String theory ${ }^{52}$, which maps the large- $N$ limit of certain conformal field theories in $d$ dimensions onto a classical theory of waves moving in the gravitational field of a black hole in a higher-dimensional curved space (Anti-de Sitter space). The conjecture can be written in a deceptively over-simplified form as

$$
\begin{aligned}
\left\langle\mathrm{T} \exp \left[\int \mathrm{d}^{d} x \phi_{0}(x) \hat{\psi}(x)\right]\right\rangle_{Q F T} & =e^{-S_{\text {grav }}\left[\phi_{0}\right]} \\
\lim _{r \rightarrow \infty} \phi_{E}(r ; x) & \rightarrow \phi_{0}(x)
\end{aligned}
$$

where $\phi_{0}(x)$ is a source term that couples to the physical fields in the $d$-dimensional field theory, while in the $(d+1)$-dimensional gravity theory, $\phi_{E}(r, x)$ is a classical field whose asymptotic $(r \rightarrow \infty)$ behaviour converges to the value $\phi_{0}(x)$.

This approach holds the mathematically tantalizing promise that by solving radial one-particle wave equations in the higher-dimensional world (albeit rather complex radial equations, for particles in the curved space-time of a charged black hole), one can extract the universal, i.e. quantum critical, physics of interacting fermions in our lower-dimensional world. The method has already shown its utility in deriving properties of certain model non-Fermi liquids with a critical Fermi 
surface $^{\underline{53}} \underline{-55}$. Nevertheless, there are there are a number of important hurdles to be overcome before this approach proves its usefulness to heavy fermion physics, in particular:

$\diamond$ In the current theories, the fermions in the "strange metal" produced at the boundary of the Anti-de Sitter space are spinless - with a purely orbital coupling to magnetic fields and consequently, a purely diamagnetic magnetic $(\chi<0)$ susceptibility. Can the Zeeman/spin physics, with a proven Pauli paramagnetism $(\chi>0)$ be impliemented in future implementations of the AdS/CFT correspondence?( One idea, suggested in $\underline{56}$ is to introduce an additional global symmetry. )

$\diamond$ There is currently no Fermi liquid solution to the AdS/CFT gravity equations. If the quantum critical behaviour seen in condensed matter physics is universal, we expect that the cross-over from a critical metal to a Fermi liquid is part of that universal theory. This should motivate future attempts to drive a cross-over to Fermi liquid behaviour in the AdS/CFT approach.

We should like to acknowledge fruitful discussions connected with this work with Vic Alexandrov, Meigan Aronson, Jeroen Custers, Rebecca Flint, Hilbert von Lohneysen, Silke Paschen, Hong Liu, Frank Steglich, Matt Strassler, Scott Thomas and Qimiao Si. This work was supported by NSF grant DMR 0907179.

1 S. Doniach, Physica B \& C 91, 231 (1977).

2 P. Gegenwart, Q. Si, and F. Steglich, Nature Physics 4, 186 (2008).

3 P. Coleman, C. Pépin, Q. Si, and R. Ramazashvili, J. Phys.: Condens. Matter 13, R723 (2001).

4 Y. Tokiwa, P. Gegenwart, C. Geibel, and F. Steglich, J. Phys. Soc. Jpn. 78, 123708 (2009).

5 S. Friedemann, T. Westerkamp, M. Brando, N. Oeschler, S. Wirth, P. Gegenwart, C. Krellner, C. Geibel, and F. Steglich, Nature Physics 5, 465 (2009).

6 J. Custers, P. Gegenwart, C. Geibel, F. Steglich, P. Coleman, and S. Paschen, Phys. Rev. Lett. 104, $186402(2010)$.

7 P. G. Niklowitz, G. Knebel, J. Flouquet, S. L. Bud'ko, and P. C. Canfield, Phys. Rev. B 73, 125101 (2006).

8 S. Nakatsuji, K. Kuga, Y. Machida, T. Tayama, T. Sakakibara, Y. Karaki, H. Ishimoto, S. Yonezawa, Y. Maeno, E. Pearson, et al., Nature Phys. 4, 603 (2008).

9 Y. Matsumoto, S. Nakatsuji, K. Kuga, Y. Karaki, N. Horie, Y. Shimura, T. Sakakibara, A. H. Nevidomskyy, and P. Coleman, unpublished (2010). 
10 H. von Löhneysen, T. Pietrus, G. Portisch, H. G. Schlager, A. Schroeder, M. Sieck, and T. Trappmann, Phys. Rev. Lett. 72, 3262 (1994).

11 H. von Löhneysen, J. Phys. Cond. Mat. 8, 9689 (1996).

12 R. Küchler, N. Oeschler, P. Gegenwart, T. Cichorek, K. Neumaier, O. Tegus, C. Geibel, J. A. Mydosh, F. Steglich, L. Zhu, et al., Phys. Rev. Lett 91, 066405 (2003).

13 S. Burdin, D. R. Grempel, and A. Georges, Phys. Rev. B 66, 045111 (2002).

14 Q. Si, Physica B 378-380, 23 (2006).

15 E. Lebanon and P. Coleman, Phys. Rev. B 76, 085117 (2007).

16 M. Vojta, Phys. Rev. B 78, 144508 (2008).

17 T. T. Ong and B. A. Jones, Phys. Rev. Lett. 103, 066405 (2009).

18 J. Custers, P. Gegenwart, C. Geibel, F. Steglich, P. Coleman, and S. Paschen, Phys. Rev. Lett. 104, $186402(2010)$.

19 S. Nakatsuji, Y. Machida, Y. Maeno, T. Tayama, T. Sakakibara, J. van Duijn, L. Balicas, J. N. Millican, R. T. Macaluso, and J. Y. Chan, Phys. Rev. Lett. 96, 087204 (2006).

20 M. S. Kim, M. C. Bennett, and M. C. Aronson, Phys. Rev. B 77, 144425 (2008).

21 M. Neumann, J. Nyeki, B. Cowan, and J. Saunders, Science 317, 1356 (2007).

22 P. W. Anderson, Phys. Rev. 86, 694 (1952).

23 P. W. A. P. Fazekas, Philosophical Magazine 30, 423 (1974).

24 B. S. Shastry and B. Sutherland, Physica B (Amsterdam) 108, 1069 (1981).

25 A. W. Sandvik, S. Daul, R. R. P. Singh, and D. J. Scalapino, Phys. Rev. Lett. 89, 247201 (2002).

26 J. Rech, P. Coleman, G. Zarand, and O. Parcollet, Phys. Rev. Lett. 96, 016601 (2006).

27 E. Lebanon and P. Coleman, Phys. Rev. B 76, 085117 (2007).

28 P. Coleman and N. Andrei, J. Phys. Cond. Matt. 1, 4057 (1989).

29 J. A. Hertz, Phys. Rev. B 14, 1165 (1976).

30 A. J. Millis, Phys. Rev. B 48, 7183 (1993).

31 Q. Si, Physica Status Solidi (b) 247, 476 (2010).

32 M. S. Kim, Bulletin of the American Physical Society p. T2.200002 (2010).

33 Z. Weihong, C. J. Halmer, and J. Oitmaa, Phys. Rev. B 60, 6608 (1999).

34 E. Müller-Hartmann, R. R. P. Singh, C. Knetter, and G. S. Uhrig, Phys. Rev. Lett. 84, 1808 (2000).

35 A. Isacsson and O. F. Syljuasen, Phys. Rev. E 74, 026701 (2006).

36 M. Albrecht and F. Mila, Europhys. Lett. 34, 145 (1996).

37 A. Koga and N. Kawakami, Phys. Rev. Lett. 84, 4461 (2000).

38 C. H. Chung, J. B. Marston, and S. Sachdev, Phys. Rev. B 64, 134407 (2001).

39 M. A. Hajj and J.-P. Malrieu, Phys. Rev. B 72, 094436 (2005).

40 T. Park, V. A. Sidorov, F. Ronning, J.-X. Zhu, Y. Tokiwa, H. Lee, E. D. Bauer, R. Movshovich, J. L. Sarrao, and J. D. Thompson, Nature 456, 366 (2008).

41 M. Nicklas, O. Stockert, T. Park, K. Habicht, K. Kiefer, L. D. Pham, J. D. Thompson, Z. Fisk, and 
F. Steglich, Phys. Rev. B 76, 052401 (2007).

42 O. Trovarelli, C. Geibel, S. Mederle, C. Langhammer, F. M. Grosche, P. Gegenwart, M. Lang, G. Sparn, and F. Steglich, Phys. Rev. Lett. 85, 626 (2000).

43 S. Paschen, T. Lühmann, S. Wirth, P. Gegenwart, O. Trovarelli, C. Geibel, F. Steglich, P. Coleman, and Q. Si, Nature 432, 881 (2004).

44 H. Shishido, R. Settai, H. Harima, and Y. Ônuki, J. Phys. Soc. Jpn. 74, 1103 (2005).

45 T. Senthil, M. Vojta, and S. Sachdev, Phys. Rev. B 69, 035111 (2004).

46 T. Senthil, Phys. Rev. B 78, 035103 (2008).

47 I. Paul, C. Pepin, and M. R. Norman, Phys. Rev. Lett. 98, 026402 (2007).

48 C. Pepin, Phys. Rev. Lett. 98, 176401 (2007).

49 C. Pepin, Phys. Rev. B 77, 245129 (2008).

50 Q. Si, S. Rabello, K. Ingersent, and J. L. Smith, Nature 413, 804 (2001).

51 Q. Si, S. Rabello, K. Ingersent, and J. L. Smith, Phys. Rev. B 68, 115103 (2003).

52 J. Maldacena, Adv. Theor. Math. Phys. 2, 231 (1998).

53 H. Liu, J. McGreevy, and D. Vegh, arXiv:0903.2477 (2009).

54 M. Cubrovic, J. Zaanen, and K. Schalm, Science 319, 1295 (2008).

55 J. McGreevy, arXiv hep-th (2009), 0909.0518v2.

56 N. Iqbal, H. Liu, M. Mezei, and Q. Si, arXiv:1003.0010 (2010). 